Pacific Journal of Mathematics

PREASSIGNNG THE SHAPE OF A FAC 


\section{PREASSIGNING THE SHAPE OF A FACE}

\section{David Barnette and Branko Grünbaum}

Theorem. Let $G$ be a 3-connected planar graph, $F$ a nonseparating $n$-circuit of $G$ with nodes $A_{1}, \cdots, A_{n}$ (in a cyclic order of $F$ ), and let $F^{\prime}$ be a convex $n$-gon with vertices $A_{1}^{\prime}, \cdots, A_{n}^{\prime}$ (in cyclic order). Then there exists a 3-polytope $P$ which realizes $G$, such that $F^{\prime}$ is a face of $P$ and that the vertex $A_{i}^{\prime}$ of $P$ corresponds to the node $A_{i}$ of $G$ for $i=1,2, \cdots, n$.

A remarkable theorem of Steinitz (see [2, p. 77], [3, p. 192], [1, p. 235]) asserts that a graph $G$ is realizable by a 3-polytope (i.e., isomorphic to the graph of the vertices and edges of a 3-dimensional convex polytope $P$ ) if and only if $G$ is planar and 3-connected. Moreover, this realization is unique up to combinatorial equivalence of the 3 -polytopes, the boundaries of the 2 -faces of each polytope realizing $G$ being determined by nonseparating circuits in $G$. (The regions of the plane determined in any imbedding of $G$ in the plane by the nonseparating circuits of $G$ will be called faces of $G$.)

Combinatorially equivalent polytopes may-intuitively speakinghave various shapes. Among the different problems concerning the freedom of choice of 3-polytopes realizing a given graph $G$, one may ask whether the shape of one $n$-gonal 2 -face $F$ of $P$ can be required to be any preassigned (convex) $n$-gon $F^{\prime}$ (see [1, p. 244]). The solution of this problem is trivially affirmative if $F$ is a triangle or a quadrangle, since in this case there exists an affine, respectively permissible for $P$ projective transformation of any realization $P$ of $G$ carrying $F$ onto any predetermined $F^{\prime}$. However, the problem ceases to be trivial already for a pentagonal face $F$.

It is the aim of the present note to establish the affirmative solution of the above problem for any face $F$. More precisely, we shall prove the theorem enunciated at the outset. By the remark made above, we shall without loss of generality assume that $n \geqq 5$.

The proof of the theorem consists mainly of a repetition of the proof of Steinitz's theorem as given in [1, pp. 236-242], with modifications resulting from the desire to interfere with $F$ as little as possible and, if such interference is unavoidable, to conduct it under special precautions. Since a repetition of the whole argument would lead to a needless duplication, we shall restrict ourselves to a summary of the proof of Steinitz's theorem, indicating the main ideas and the necessary changes in the argumentation. We shall use the terminology and notation employed in [1].

The proof of Steinitz's theorem proceeds by induction on the 
number of edges of $G$, and two cases have to be considered. In each case, the graph $G$ is "reduced" to a 3-connected planar graph $G^{*}$ by a change of such a nature that from each realization of $G^{*}$ by a 3-polytope $P^{*}$ a realization of $G$ by a 3-polytope $P$ may be constructed. The "reductions" needed for the proof are indicated in Figure 1.

Case 1. The graph contains a nonseparating circuit with three edges (i.e., a triangular face, or triangle) one node of which has valence 3 . Then one of the reductions $\eta_{1}, \eta_{2}, \eta_{3}$ may be applied, yielding a $G^{*}$ with 1,2 , or 3 edges less than $G$. In the proof of Steinitz's theorem, there is nothing to be added in this case, since induction takes over. In the proof of our theorem one has to require that $G^{*}$ be realized by a 3-polytope $P^{*}$ having $F^{\prime}$ as a face, unless the circuit $F$ contains one of the edges-marked by one, two, or three stars in Figure 1-of the triangle in question. In those exceptional cases the circuit $F^{*}$ of $G^{*}$ (corresponding to the circuit $F$ of $G$, and having also $n$ edges in cases denoted by one star, $n-1$ edges in cases denoted by two stars, and $n+1$ edges in cases denoted by three stars) has to correspond in $P^{*}$ to a face $F^{* \prime}$ with vertices $A_{1}^{* \prime}, A_{2}^{* \prime}, \ldots$ different from $F^{\prime}$. The construction of the polygon $F^{* \prime}$ from $F^{\prime}$ is indicated
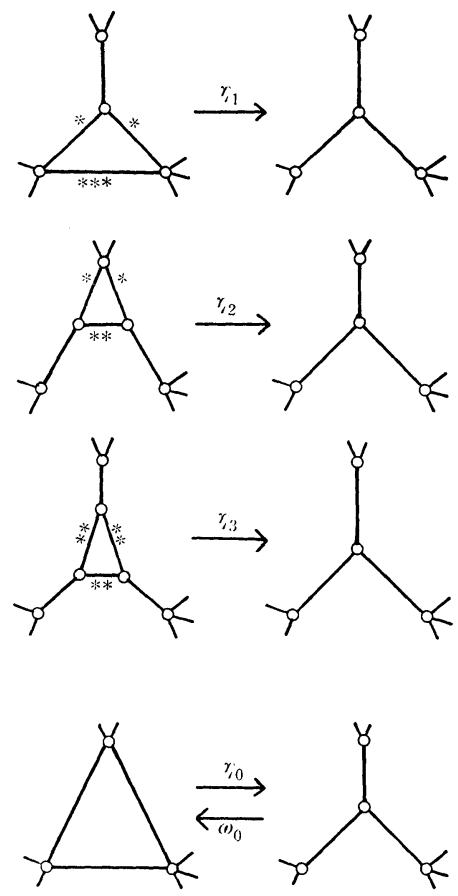

Figure 1 

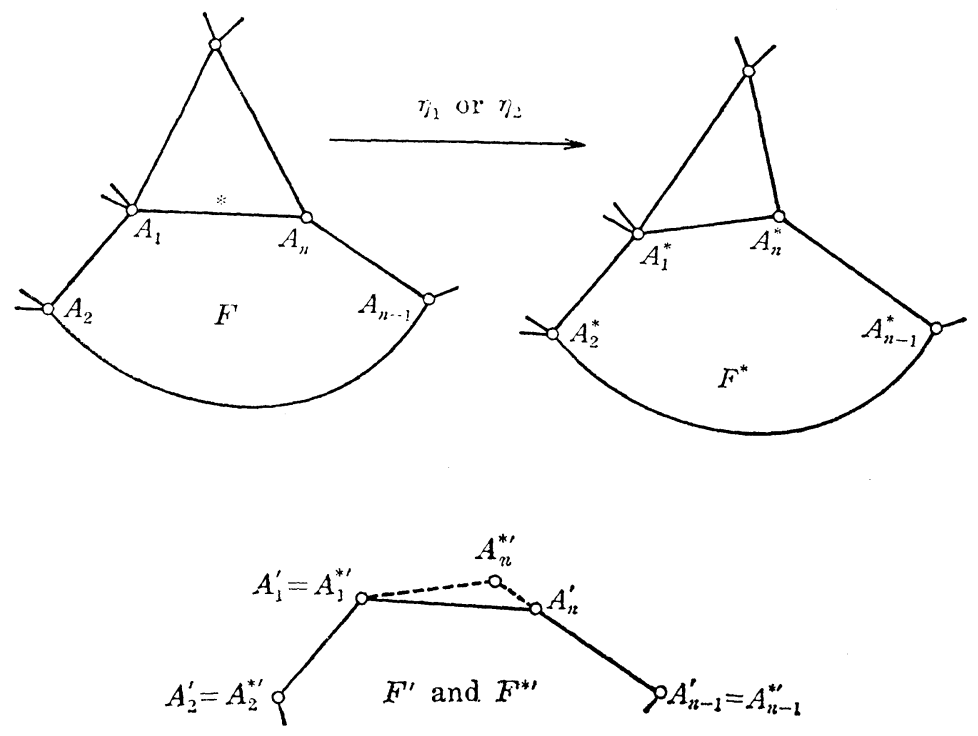

FiguRE 2
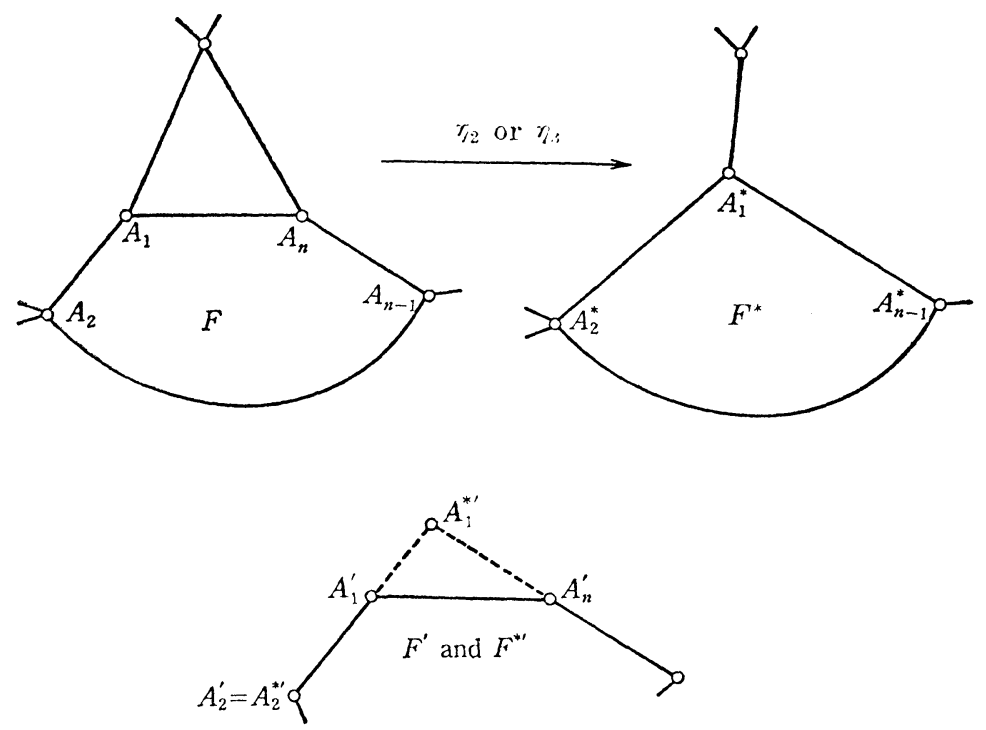

FIGURE 3

in Figures 2 and 3 for the first two cases; in case of the edge denoted by three stars, $F^{* \prime}$ may be any $(n+1)$-gon obtained as the convex hull of $F^{\prime}$ and a point $A_{n+1}^{* \prime}$ near the edge $A_{n}^{\prime} A_{1}^{\prime}$. (Note that in the case represented in Figure 3 it may be necessary to start not with $F^{\prime}$ itself but-in order to guarantee the existence of $F^{* \prime}$-with a suitable projective transformation of $F^{\prime}$. However, this does not impair the construction since a suitable inverse projective transformation of the polytope realizing $G$ will restore $F^{\prime}$ as a face of $P^{\prime}$.) 
This completes the proof in Case 1 .

Case 2. Now we assume that $G$ contains no triangle possessing at least one 3-valent node. Since in any case Euler's relation implies that $G$ contains either a triangle, or a 3 -valent vertex, it follows that now one of the reductions $\omega_{0}$ or $\eta_{0}$ may be applied to $G$. However, those reductions do not reduce the number of edges and may thus be deemed useless. The depth of Steinitz's proof lies in establishing that by a judicious choice of a finite sequence of $\omega_{0}$ or $\eta_{0}$ reductions (not exceeding in total number the number of edges of $G$ ) one may transform $G$ into a graph $\widetilde{G}$ which is covered by Case 1 . In order to prove our theorem we shall show that there is enough freedom in the choice of the $\omega_{0}$ and/or $\eta_{0}$ reductions to reach $\widetilde{G}$ without interfering (in any of the stages) with the circuit $F$ of $G$ (or the corresponding circuits in the intermediate stages). The derived realization of $\widetilde{G}$ and of all the intermediate graphs will still have $F^{\prime}$ as the preassigned polygon.

Before proving those assertions, we have to introduce a number of notions. For each 3-connected planar graph $G$, we define a graph $I(G)$ as follows. The vertices of $I(G)$ correspond to edges of $G$; two vertices of $I(G)$ are connected by an edge if and only if the corresponding edges of $G$ have a common node and belong to the same nonseparating circuit. (In Figure 4, a graph $G$ is shown by heavy lines, while $I(G)$ is indicated by thin lines.) It is easily seen that $I(G)$ if planar and 3-connected. Note that $I(G)$ is 4-valent, and that each $n$-gonal region of $I(G)$ corresponds either to an $n$-gonal region of $G$ or to an $n$-valent vertex of $G$. A "geodesic arc" in $I(G)$ is a path in $I(G)$ in which each two adjacent edges separate the other two edges of $I(G)$ issuing from their common node. A "lens" of $I(G)$ is

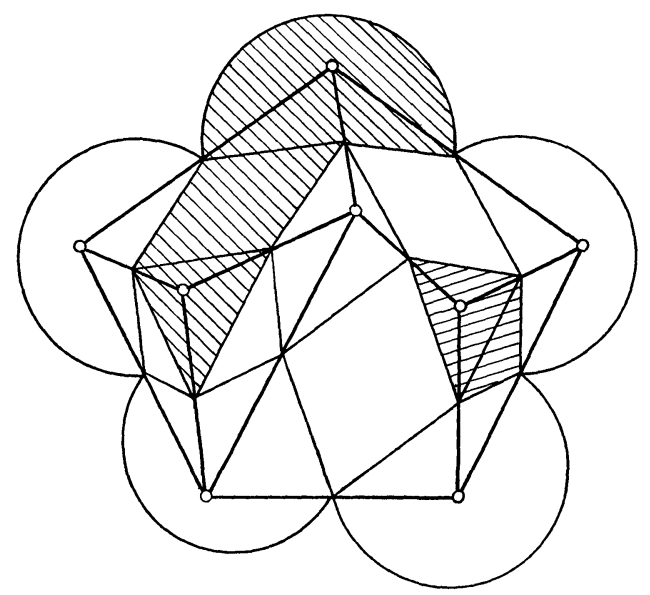

FiguRE 4 
a region of plane having as boundary two geodesic arcs of $I(G)$, such that at their common endpoints (the "poles" of the lens) the remaining two edges of $I(G)$ do not belong to the lens. (For example, each of the shaded regions in Figure 4 is a lens, one with poles $A$ and $B$, the other with poles $C$ and $D$; the second lens is clearly the simplest possible type, consisting of only two triangles; we shall denote it by $L_{0}$.)

An important role is played by "irreducible lenses", that is, lenses which do not contain any proper sublenses. The relevant facts about irreducible lenses are (see [1, pp. 239-242]):

(1) Each region of the plane which has a boundary consisting either of a (closed) geodesic arc, or of two geodesic arcs, contains an irreducible lens. This implies, among other consequences, the existence of irreducible lenses in each $I(G)$.

(2) Each irreducible lens contains at least two triangles incident to the boundary of the lens; unless the lens is $L_{0}$, they have no edge in common.

(3) If an irreducible lens $L \neq L_{0}$ of $I(G)$ is given, and if an $\omega_{0}$ or $\eta_{0}$ reduction is performed on a 3 -valent node or on a triangle of $G$ corresponding to a triangle of $L$ having an edge in the boundary of $L$, the resulting graph $G^{*}$ has the following property: There is a lens (and hence, by (1), an irreducible lens $L^{*}$ ) of $I\left(G^{*}\right)$ having fewer faces than $L$, such that its faces correspond to some of the faces of $L$.

Combining (1), (2) and (3) the proof of Steinitz's theorem is completed by starting from any region bounded by one or two geodesic arcs, finding an irreducible lens $L$ in it, and applying a suitable $\omega_{0}$ or $\eta_{0}$ reduction. Since $L$ contains only finitely many faces, by repeated application of (3) we necessarily reach a graph $G$ containing a lens of type $L_{0}$. But the presence of such a lens in $I(G)$ means that $G$ contains a triangle with a trivalent node; hence to $G$ the Case 1 is applicable. (In the proof of Steinitz's theorem $\mathrm{L}$ is chosen to contain the least number of faces among all lenses of $I(G)$, hence there is no necessity to use the italicized part of (3); however, this part of (3) is evident from the proof of the first part [1, p. 242].)

In view of the above, in order to complete the proof of our theorem it is sufficient to exhibit, for each $G$ which is not covered by Case 1, a region $R$ bounded by one or two geodesic arcs of $I(\mathrm{G})$ and such that at most one of the triangles of $R$ corresponds to a node of $G$ belonging to $F$. Indeed, this property will be inherited by any irreducible lens $L$ contained in $R$, and hence $L$ will contain at least one other triangle such that the 3-valent node, or triangle, of $G$ corresponding to it is disjoint from $F$. Thus an appropriate reduction $\omega_{0}$ or $\eta_{0}$ will be applicable without interference with $F$, and $F^{\prime}$ may be chosen as the preassigned face of a 3-polytope realizing the reduced 


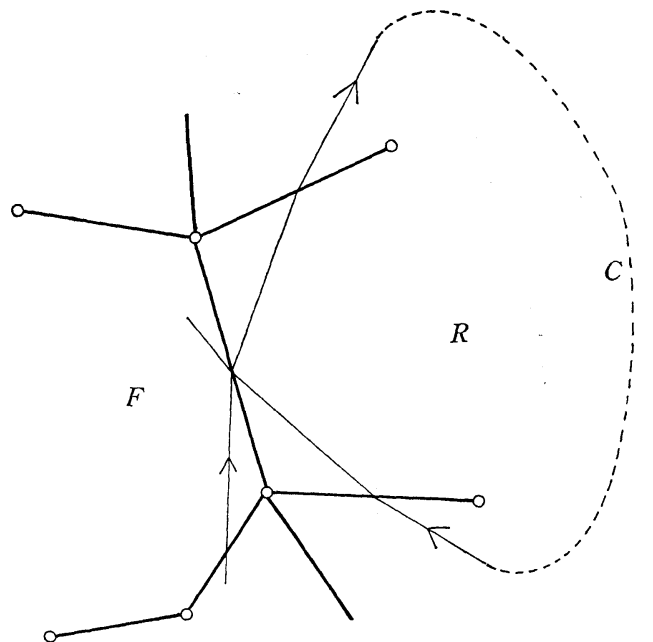

Figure 5
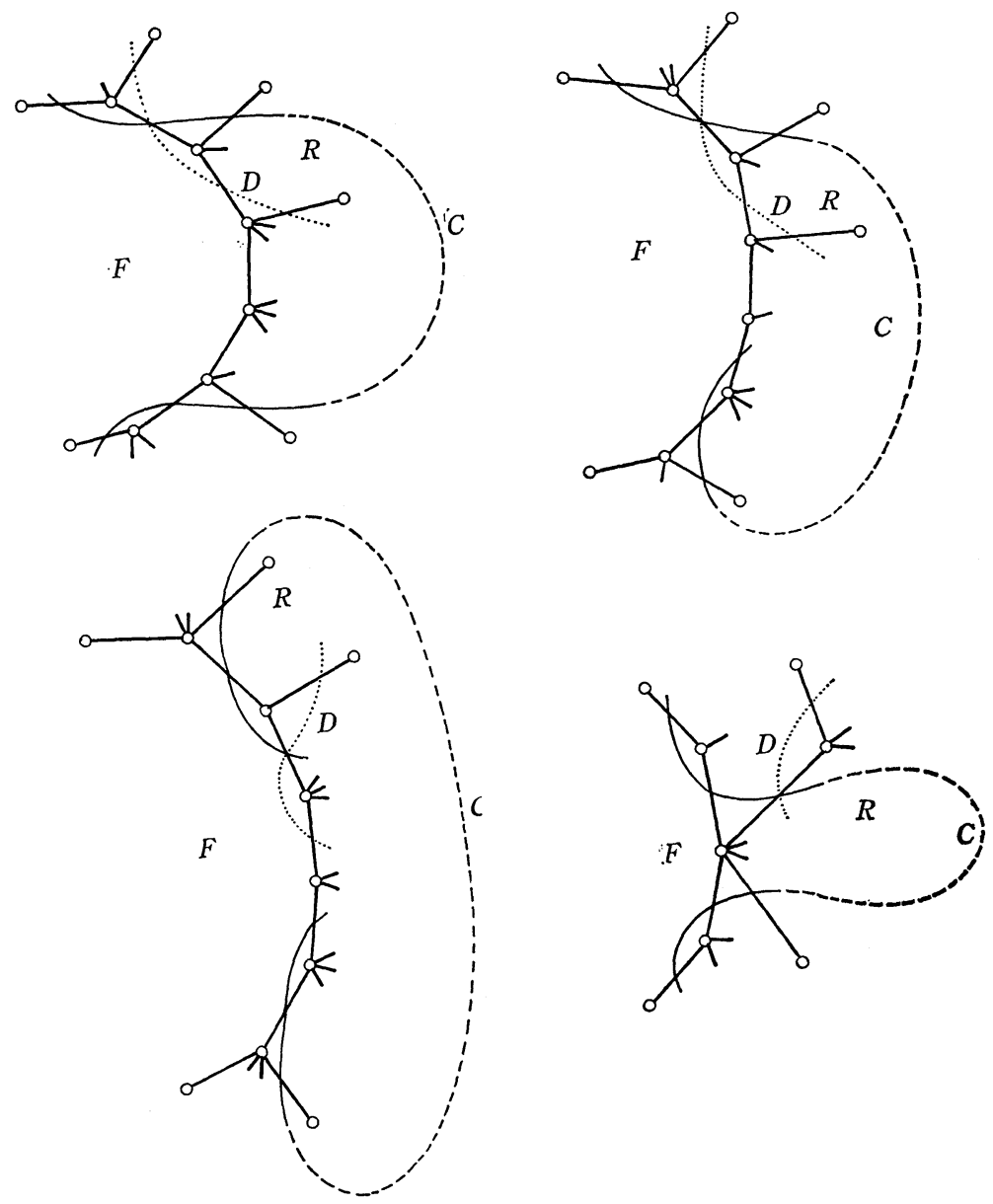

Figure 6 
graph.

But the existence of a region $R$ of the type required is easy to establish. Proceeding on any geodesic arc (starting with an edge of $I(G)$ having both endpoints on edges of $F$ ) we note that if $C$ intersects itself prior to its return to $F$, or if it self-intersects while reentering $F$, that region enclosed by $C$ which does not contain $F$ may be taken as $R$. (Compare the schematic drawing in Figure 5.) Hence we are left only with the case in which each geodesic arc is free of selfintersections outside $F$. Among all the (simple) geodesic arcs of $I(G)$ having endpoints at edges of $F$, we choose one bridging the smallest possible number of edges of $F$ and denote it by $C$. The possible situations are schematically indicated in Figure 6. In the first three cases the minimality of $C$ prevents $D$ from returning to $F$ before meeting $C$ again, and thus parts of $C$ and $D$ will determine a region $R$ of the type required. In the fourth case $D$ is any geodesic arc crossing $C$ at a relatively interior point, and $D$ either meets $C$ before meeting $F$, or it meets $C$ and $F$ at the same vertex, again producing a region of the type required.

This completes the proof in Case 2, and with it the proof of the theorem.

REMARK 1. As an easy corollary of the theorem we have:

If $P$ is a 3-polytope and if $C$ is a simple closed circuit of edges of $P$ such that no facet of $P$ meets two edges of $C$, there exists a polytope $P^{\prime}$ combinatorially equivalent to $P$ such that the circuit of $P^{\prime}$ corresponding to $C$ is in a plane.

2. By an obvious application of duality, if follows from the theorem that the shape of one vertex-figure of a 3-polytope may be arbitrarily prescribed. Probably, more elements of a 3-polytope may be arbitrarily prescribed; however, it is easy to see that it is not always possible to preassign the shape of two faces having a common edge. (For example, the two quadrilaterals of Figure 7 may not appear in any 3 -sided prism.) It would be interesting to investigate the following

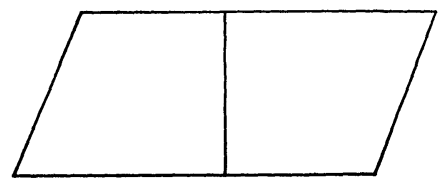

FIGURE 7

Conjecture. For any family $\left\{F_{1}, \cdots, F_{n}\right\}$ of disjoint faces of a 3-polytope $P$, and for any family $\left\{F_{1}^{\prime}, \cdots, F_{n}^{\prime}\right\}$ of polygons, $F_{i}^{\prime}$ being 
of the same type as $F_{i}$, there exists a 3-polytope $P^{\prime}$ combinatorially equivalent to $P$, such that every face of $P^{\prime}$ corresponding to one of the faces $F_{i}$ is projectively equivalent to $F_{i}^{\prime}$.

3. It would be very interesting to determine to what extent the theorem holds in higher dimensions. The only known result in this direction seems to be M.A. Perles' example of an 8-dimensional polytope $P$ with 12 vertices such that the shape of one of its 7-dimensional faces (with 10 vertices) may not be arbitrarily chosen within its combinatorial type $([1$, p. 96 , Exercise 3$])$. It may be conjectured that a similar failure of the theorem occurs already in four dimensions.

\section{REFERENCES}

1. B. Grünbaum, Convex Polytopes, J. Wiley and Sons, 1967.

2. E. Steinitz, Polyeder und Raumeinteilungen, Enzykl. Math. Wiss. Part 3A B12 (Geometrie),(1922), 1-139.

3. E. Steinitz and H. Rademacher, Vorlesungen über die Theorie der Polyeder, Springer, 1934.

Received January 8, 1968. Research supported by National Science Foundation grants GP-3579 and GP-7536.

UNIVERSITY OF WASHINGTON 


\section{PACIFIC JOURNAL OF MATHEMATICS}

\section{EDITORS}

\author{
H. SAMELSON \\ Stanford University \\ Stanford, California 94305 \\ RICHARD PIERCE \\ University of Washington \\ Seattle, Washington 98105
}

J. DugundJI

Department of Mathematics University of Southern California Los Angeles, California 90007

BASIL GORDON*

University of California

Los Angeles, California 90024

\section{ASSOCIATE EDITORS}
E. F. BECKENBACH
B. H. NEUMANN
F. WOLE
K. YOSHIDA

\section{SUPPORTING INSTITUTIONS}

\author{
UNIVERSITY OF BRITISH COLUMBIA \\ CALIFORNIA INSTITUTE OF TECHNOLOGY \\ UNIVERSITY OF CALIFORNIA \\ MONTANA STATE UNIVERSITY \\ UNIVERSITY OF NEVADA \\ NEW MEXICO STATE UNIVERSITY \\ OREGON STATE UNIVERSITY \\ UNIVERSITY OF OREGON \\ OSAKA UNIVERSITY \\ UNIVERSITY OF SOUTHERN CALIFORNIA
}

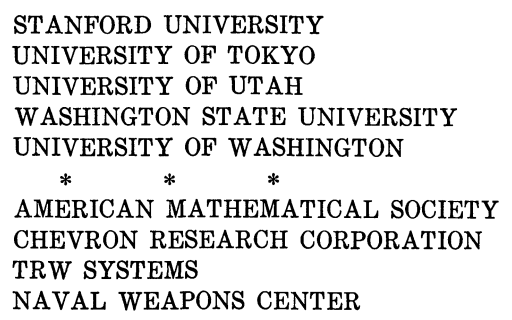

The Supporting Institutions listed above contribute to the cost of publication of this Journal, but they are not owners or publishers and have no responsibility for its content or policies.

Mathematical papers intended for publication in the Pacific Journal of Mathematics should be in typed form or offset-reproduced, (not dittoed), double spaced with large margins. Underline Greek letters in red, German in green, and script in blue. The first paragraph or two must be capable of being used separately as a synopsis of the entire paper. The editorial "we" must not be used in the synopsis, and items of the bibliography should not be cited there unless absolutely necessary, in which case they must be identified by author and Journal, rather than by item number. Manuscripts, in duplicate if possible, may be sent to any one of the four editors. Please classify according to the scheme of Math. Rev. 36, 1539-1546. All other communications to the editors should be addressed to the managing editor, Richard Arens, University of California, Los Angeles, California, 90024.

50 reprints are provided free for each article; additional copies may be obtained at cost in multiples of 50 .

The Pacific Journal of Mathematics is published monthly. Effective with Volume 16 the price per volume (3 numbers) is $\$ 8.00$; single issues, $\$ 3.00$. Special price for current issues to individual faculty members of supporting institutions and to individual members of the American Mathematical Society: $\$ 4.00$ per volume; single issues $\$ 1.50$. Back numbers are available.

Subscriptions, orders for back numbers, and changes of address should be sent to Pacific Journal of Mathematics, 103 Highland Boulevard, Berkeley, California, 94708.

PUBLISHED BY PACIFIC JOURNAL OF MATHEMATICS, A NON-PROFIT CORPORATION

Printed at Kokusai Bunken Insatsusha (International Academic Printing Co., Ltd.), 7-17, Fujimi 2-chome, Chiyoda-ku, Tokyo, Japan.

* Acting Managing Editor. 


\section{Pacific Journal of Mathematics}

\section{Vol. 32, No. $2 \quad$ February, 1970}

Harry P. Allen and Joseph Cooley Ferrar, Jordan algebras and exceptional subalgebras of the exceptional algebra $E_{6} \ldots \ldots \ldots \ldots \ldots \ldots \ldots 283$

David Wilmot Barnette and Branko Grünbaum, Preassigning the shape of a

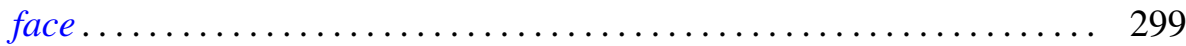

Robert Francis Craggs, Involutions of the 3-sphere which fix 2-spheres . . . . 307

David William Dean, Bor-Luh Lin and Ivan Singer, On k-shrinking and $k$-boundedly complete bases in Banach spaces ................ 323

Martin Engert, Finite dimensional translation invariant subspaces ....... 333

Kenneth Lewis Fields, On the global dimension of residue rings ......... 345

Howard Gorman, The Brandt condition and invertibility of modules ....... 351

Benjamin Rigler Halpern, A characterization of the circle and interval ..... 373

Albert Emerson Hurd, A uniqueness theorem for second order quasilinear

hyperbolic equations ............................... 415

James Frederick Hurley, Composition series in Chevalley algebras ...... 429

Meira Lavie, Disconjugacy of linear differential equations in the complex

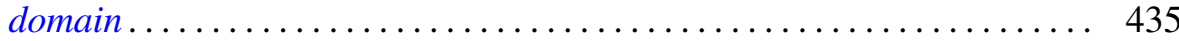

Jimmie Don Lawson, Lattices with no interval homomorphisms ......... 459

Roger McCann, A classification of center-foci ................. 467

Evelyn Rupard McMillan, On continuity conditions for functions . . . . . . . 479

Graciano de Oliveira, A conjecture and some problems on permanents .... 495

David L. Parrott and S. K. Wong, On the Higman-Sims simple group of order $44,352,000$.

Jerome L. Paul, Extending homeomorphisms ................. 517

Thomas Benny Rushing, Unknotting unions of cells .............. 521

Peter Russell, Forms of the affine line and its additive group.......... 527

Niel Shilkret, Non-Archimedean Gelfand theory ................. 541

Alfred Esperanza Tong, Diagonal submatrices of matrix maps.......... 551 